\title{
Effect of maternal anemia on fetal Doppler indices during the last trimester of pregnancy
}

\author{
Original \\ Article \\ Abdel-Megeed Ismail Abdel-Megeed, Amr Ahmed Mahmoud Riad, Shaimaa Mahmoud \\ Mohamed Morsi Elsherif
}

Department of Obstetrics and Gynecology, Faculty of Medicine, Ain Shams University, Cairo, Egypt

\begin{abstract}
Aim of the work: The aim of this study was to evaluate the effect of maternal anemia on fetal Doppler indices ; namely, umbilical artery and middle cerebral artery in the last trimester of pregnancy.

Patients and Methods: This study was designed as a prospective case control clinical trial carried out in obstetric outpatient clinics and inpatient ward, Ain Shams University Maternity Hospital on 200 patients. The patients must follow these criteria:Gestational age between 28-40 weeks of singleton pregnancy (calculated by their last menstrual period or by earlier ultrasound), fetus is alive and normal fetal ultrasound parameters.

Results: The umbilical artery resistance index showed a significant increase in moderate severe anemic patients more than the control group. Umbilical artery pulsatility index showed a significant increase in severe anemic group more than the other 3 groups.Umbilical artery systolic/diastolic ratio showed a significant increase in severe anemic more than the other groups. Finally, the umbilical artery cerebral/umbilical artery resistance ratio showed a significant increase in severe anemic more than the other groups.

Conclusion: GA at delivery in different groups was matched, i.e. there was no statistical significant difference between different studied groups regarding GA at delivery $(P>0.05)$. Neonatal ICU admission were 2(4\%), 2(4\%), 4(8\%) and $9(18 \%)$ in different groups, respectively. There was statistical significant difference between different studied groups regarding Neonatal ICU admission $(P<0.05)$.
\end{abstract}

Key Words: Cerebral/umbilical artery resistance ratio, hemoglobin concentration, red blood cells.

Received: 25 October 2018, Accepted: 11 November 2018.

Corresponding Author: Shaimaa Mahmoud Mohamed, MSc., Department of Obstetrics and Gynecology, Faculty of Medicine, Ain Shams University, Cairo, Egypt, Tel: 01024035319, Email: drshaymaa1987@yahoo.com.

ISSN: 2090-7265, February 2019, Vol.9, No.1

\section{INTRODUCTION}

Anemia in general is characterized by a decrease in number of red blood cells or less than the normal quantity of hemoglobin. The condition is determined by the expected normal range of hemoglobin in a population, and is defined as existing in an individual whose hemoglobin concentration $(\mathrm{Hb})$ has fallen below a threshold lying at two standard deviations below the median for a healthy population of the same demographic characteristics, including age, sex and pregnancy status ${ }^{[1]}$.

The actual process of making red blood cells is called erythropoiesis. In Greek, erythro means "red," and poiesis means "the making of things." So, Erythropoiesis is the process of manufacturing, recycling, and regulating the number of red blood cells ${ }^{[2]}$. Most of the work of erythropoiesis occurs in the bone marrow. It takes about 25 days. In children younger than 5 years old, the marrow of all bones of the body is enlisted for producing red blood cells. As a person ages, red blood cells are eventually produced only in the marrow of the spine, ribs, and pelvis ${ }^{[3]}$.

According to the United Nations (UN) estimates, approximately half of pregnant women suffer from anemia worldwide. Nutritionally related iron deficiency is the main cause of anemia throughout the world. It is especially common in women of reproductive age and particularly during pregnancy. The demand for iron increases about six to seven times from early pregnancy to the late pregnancy ${ }^{[4]}$.

Folate deficiencies since 1960s and B12 vitamin deficiencies since 1990s were defined as contributing causes of nutritional anemia. Folate deficiency is common as a result of dietary deficiency or increased demand as in pregnancy. The prevalence of folate deficiency in pregnant patients varies from 1 to $50 \%$. The prevalence is higher among economically deprived patients. B12 vitamin is mostly found in food of animal origin and deficiency was not reported as a frequent cause of anemia. Anemia due to zinc, copper, vitamin A or other vitamin and minerals are also considered in the literature ${ }^{[4]}$. 


\section{AIM OF THE STUDY}

This study aims to evaluate the effect of maternal anemia on fetal Doppler indices ; namely, umbilical artery and middle cerebral artery in the last trimester of pregnancy.

\section{PATIENTS AND METHODS}

Study Design: This study was designed as a prospective case control clinical trial.

Study Site: Obstetric outpatient clinics and inpatient ward, Ain Shams University Maternity Hospital.The study was approved by the Ethics Board of Ain Shams University and an informed written consent was taken from each participant in the study.

Duration of the study: The study was done during the period between January 2017 and January 2018.

Sample Size: Total sample size in the study was calculated to be two hundred patients (200). They were randomized into two groups:

1-Group one: anemic pregnantfemales (study group) including 150 women divided according to degree of anemia. Group two: non anemic pregnant females (control group) including 50 women.

2-Study population: Study population comprises women attending Ain Shams Maternity Hospital,the obstetric outpatient clinics and inpatient ward, fulfilling the inclusion criteria required for the study and who agreed to participate after consent approval.

Subject: These pregnant ladies were divided into two groups:

1- Study Group : (anemia group) including 150 women divided according to degree of anemia into three subgroups : Mild anemia: including 50 women with hemoglobin level 10-10,9 g/dl. Moderate anemia: including 50 women with hemoglobin level 7-9,9 g/dl. Severe anemia: including 50 women with hemoglobin level lower than $7 \mathrm{~g} \backslash \mathrm{dl}$.

2-Control Group: including 50 women with hemoglobin level equal or above $11 \mathrm{~g} / \mathrm{dl}$.

A"Mild" is a misnomer: iron deficiency is already advanced by the time anemia is detected. The deficiency has consequences even when no anemia is clinically apparent. The hemoglobin cut-off of $11 \mathrm{~g} / \mathrm{dl}$ for pregnant women was first presented in the 1968. In healthy, iron-sufficient women, hemoglobin concentrations change dramatically during pregnancy to accommodate the increasing maternal blood volume and the iron needs of the fetus. Concentrations decline during the first trimester, reaching their lowest point in the second trimester, and begin to rise again in the third trimester.

With the following criteria for both groups: Inclusion criteria included gestational age between 28-40 weeks of singleton pregnancy (calculated by their last menstrual period or by earlier ultrasound) ; Fetus is alive ; Normal fetal ultrasound parameters. Exclusion criteria included experiencing labor, any major congenital fetal anomalies, any hemoglobinopathies, hemolytic anemia, anemia of chronic infection, intrauterine fetal growth retardation, oligohydramnios or polyhydramnios and maternal diseases like diabetes, pre-eclampsia, bronchial asthma, etc.

Data Management and Analysis:The collected data has been revised, coded, tabulated and introduced to a PC using Statistical package for Social Science (SPSS 15.0.1 for windows; SPSS Inc, Chicago, IL, 2001). Data were presented as mean and standard deviation $( \pm \mathrm{SD})$ for quantitative parametric data and median and interquartile range for quantitative non-parametric data. Frequency and percentage were used for presenting qualitative data. Suitable analysis was done according to the type of data obtained. Student T Test or Mann Whitney test was used to analyze quantitative data ; while Chisquare test and fisher exact test were used to analyze qualitative data. $P$ - value: level of significance: $P>0.05$ : Non-significant (NS). $-P<0.05$ : Significant (S). $P<0.01$ : Highly significant (HS).

\section{RESULTS}

Table 1 shows comparison between the different studied groups regarding demographic data. Age in non-anemia group ranged between $22-35$ with mean value $28.7 \pm 3.76$ and in anemia group ranged from $22-35$ with mean value $28.5 \pm 4.23$

Parity in non-anemia group ranged from $0-3$ child with mean value $1.5 \pm 1.05$ and was ranged from $0-3$ child in anemia group with mean value $1.4 \pm 1.12$. Hemoglobin in non-anemia group ranged from 11-13.8 with mean value $12.4 \pm 0.91$ and ranged from 5-10.9 in anemia group with mean value $8.3 \pm 1.93$. Gestational age at the Doppler in non-anemia group ranged from $28-40$ with mean value $34.1 \pm 3.75$ and in anemia group ranged from 28-40 with mean value $33.7 \pm 3.48$. Gestational age at delivery in non-anemia group ranged from $38-40$ with mean value $40.0 \pm 1.29$ nd in anemia group ranged from $38-40$ with mean value $40.1 \pm 1.43$. 
Table 1: Comparison between the different studied groups regarding demographic data

\begin{tabular}{|c|c|c|c|c|}
\hline & Non anemia group & Anemia group & $\mathrm{t}$-test & $P$ \\
\hline \multicolumn{5}{|c|}{ Age (years) } \\
\hline Range & $22.00-35$ & $22-35$ & \multirow{3}{*}{0.956} & (A) 212 \\
\hline Mean & 28.7 & 28.5 & & $\begin{array}{l}0.313 \\
\mathrm{NS}\end{array}$ \\
\hline $\mathrm{SD}$ & 3.76 & 4.23 & & \\
\hline \multicolumn{5}{|l|}{ Parity } \\
\hline Range & $0-3$ & $0-3$ & \multirow{3}{*}{0.89} & 0280 \\
\hline Mean & 1.5 & 1.4 & & $\begin{array}{c}0.289 \\
\mathrm{NS}\end{array}$ \\
\hline $\mathrm{SD}$ & 1.05 & 1.12 & & \\
\hline \multicolumn{5}{|c|}{ Hemoglobin $(\mathrm{gm} / \mathrm{dl})$} \\
\hline Range & $11-13.8$ & $5-10.9$ & \multirow{3}{*}{5.32} & $0001 *$ \\
\hline Mean & 12.4 & 8.3 & & $\begin{array}{l}0.001 \\
\mathrm{HS}\end{array}$ \\
\hline SD & 0.91 & 1.93 & & \\
\hline \multicolumn{5}{|c|}{ Gestational age at which the Doppler was performed in weeks } \\
\hline Range & $28-40$ & $28.0-40$ & \multirow{3}{*}{1.65} & \\
\hline Mean & 34.1 & 33.7 & & $0.0 / 0$ \\
\hline $\mathrm{SD}$ & 3.75 & 3.48 & & \\
\hline \multicolumn{5}{|c|}{ Gestational age at delivery in weeks } \\
\hline Range & $38-42$ & $38-42$ & \multirow{3}{*}{.98} & \multirow{3}{*}{$\begin{array}{l}0.36 \\
\mathrm{NS}\end{array}$} \\
\hline Mean & 40.0 & 40.1 & & \\
\hline SD & 1.29 & 1.43 & & \\
\hline
\end{tabular}

Data are expressed as mean $\pm \mathrm{SD}$

Table 2 shows comparison between the different studied groups regarding Middle cerebral artery resistance index, pulsatility index, systolic/diastolic ratio and peak systolic velocity. Middle cerebral artery resistance index in control group ranged from 0.792-0.923 with mean value $0.821 \pm 0.035$, in mild anemia group ranged from $0.78-0.98$ with mean value $7.34 \pm 0.06$, in moderate anemia group ranged from $0.79-0.91$ with mean value $0.81 \pm 0.06$, in severe anemia group ranged from $0.56-0.80$ with mean value $0.70 \pm 0.08$.

There was statistically significant relation between the different studied groups regarding Middle cerebral artery resistance index $(P<0.05)$.

In control group, middle cerebral artery pulsatility index ranged from 1.42-1.71 with mean value $1.42 \pm 0.10$, in mild anemia group ranged from 1.15-1.75 with mean value $1.41 \pm 0.18$, in moderate anemia group ranged from 1.13-1.71 with mean value $1.42 \pm 0.18$, in severe anemia group ranged from 1.40-1.84with mean value $1.56 \pm 0.13$. There was statistically significant relation between the different studied groups regarding Middle cerebral artery pulsatility index $(P<0.05)$. In control group, middle cerebral artery systolic/diastolic ratio ranged from 2.99-4.81 with mean value $3.82 \pm 0.51$, in mild anemia group ranged from 2.79-4.77 with mean value $3.86 \pm 0.61$, in moderate anemia group ranged from $2.77-4.70$ with mean value $3.64 \pm 0.62$, in severe anemia group ranged from 2.61-4.17 with mean value $3.33 \pm 0.49$.

There was statistically significant relation between the different studied groups regarding Middle cerebral artery systolic/diastolic ratio $(P<0.05)$. In control group, middle cerebral artery peak systolic velocity ranged from 31-51 with mean value $42.24 \pm 6.65$, in mild anemia group ranged from $31.7-50.60$ with mean value $40.42 \pm 5.65$, in moderate anemia group ranged from 30.30-50.50 with mean value $40.57 \pm 5.35$, in severe anemia group ranged from $30-52$ with mean value $42.11 \pm 6.21$.

There was no statistically significant relation between the different studied groups regarding middle cerebral artery peak systolic velocity $(P>0.05)$. 
Table 2: Comparison between the different studied groups regarding Middlecerebral artery resistance index, pulsatility index, systolic/ diastolic ratio and peak systolic velocity.

\begin{tabular}{|c|c|c|c|c|}
\hline & Control group & Mild Anemia & Moderate Anemia & Severe anemia \\
\hline \multicolumn{5}{|c|}{ Middle cerebral artery resistance index } \\
\hline $\begin{array}{l}\text { Range } \\
\text { Mean } \\
\text { S.D. }\end{array}$ & $\begin{array}{c}0.792-0.923 \\
0.821 \\
0.035\end{array}$ & $\begin{array}{c}0.78-0.98 \\
7.34 \\
0.06\end{array}$ & $\begin{array}{c}0.79-0.91 \\
0.81 \\
0.06\end{array}$ & $\begin{array}{c}0.56-0.80 \\
0.70 \\
0.08\end{array}$ \\
\hline $\begin{array}{l}\mathrm{F} \\
\mathrm{P}\end{array}$ & \multicolumn{4}{|c|}{$\begin{array}{c}8.21 \\
0.016^{*}\end{array}$} \\
\hline \multicolumn{5}{|c|}{ Middle cerebral artery pulsatility index } \\
\hline $\begin{array}{l}\text { Range } \\
\text { Mean } \\
\text { S.D. }\end{array}$ & $\begin{array}{c}1.42-1.71 \\
1.42 \\
0.10\end{array}$ & $\begin{array}{c}1.15-1.75 \\
1.41 \\
0.18\end{array}$ & $\begin{array}{c}1.13-1.71 \\
1.42 \\
0.18\end{array}$ & $\begin{array}{c}1.40-1.84 \\
1.56 \\
0.13\end{array}$ \\
\hline $\begin{array}{l}\mathrm{F} \\
\mathrm{P}\end{array}$ & \multicolumn{4}{|c|}{$\begin{array}{c}7.10 \\
0.021 *\end{array}$} \\
\hline \multicolumn{5}{|c|}{ Middle cerebral artery systolic/diastolic ratio } \\
\hline $\begin{array}{l}\text { Range } \\
\text { Mean } \\
\text { S.D. }\end{array}$ & $\begin{array}{c}2.99-4.81 \\
3.82 \\
0.51\end{array}$ & $\begin{array}{c}2.79-4.77 \\
3.86 \\
0.61\end{array}$ & $\begin{array}{l}2.77-4.70 \\
3.64 \\
0.62\end{array}$ & $\begin{array}{l}2.61-4.17 \\
3.33 \\
0.49\end{array}$ \\
\hline $\begin{array}{l}\mathrm{F} \\
\mathrm{P}\end{array}$ & \multicolumn{4}{|c|}{$\begin{array}{c}9.371 \\
0.0001 *\end{array}$} \\
\hline \multicolumn{5}{|c|}{ Middle cerebral artery peak systolic } \\
\hline $\begin{array}{l}\text { Range } \\
\text { Mean } \\
\text { S.D. }\end{array}$ & $\begin{array}{c}31.00-51.90 \\
42.24 \\
6.65\end{array}$ & $\begin{array}{c}31.70-50.60 \\
40.42 \\
5.65\end{array}$ & $\begin{array}{c}30.30-50.50 \\
40.57 \\
5.35\end{array}$ & $\begin{array}{c}30.00-52.00 \\
42.11 \\
6.21\end{array}$ \\
\hline $\begin{array}{l}\mathrm{F} \\
\mathrm{P}\end{array}$ & \multicolumn{4}{|c|}{$\begin{array}{l}1.309 \\
0.273\end{array}$} \\
\hline
\end{tabular}

\section{DISCUSSION}

Iron deficiency is defined as a condition in which there are no mobilizable iron stores, resulting from a longterm negative iron balance and leading to a compromised supply of iron to the tissues. Finally, the most significant negative consequence of ID is anemia, usually microcytic hypochromic in nature ${ }^{[5]}$.

Iron deficiency and iron deficiency anemia during pregnancy are risk factors for preterm delivery, prematurity and small for gestational age birth weight. Iron deficiency has a negative effect on intelligence and behavioral development in the infant. It is essential to prevent iron deficiency in the fetus by preventing iron deficiency in the pregnant woman ${ }^{[6]}$.

The current study aims to evaluate the effect of maternal anemia on fetal Doppler indices ; namely, umbilical artery and middle cerebral artery in the last trimester of pregnancy.

This prospective case control clinical study was carried out on 200 pregnant women classified into two groups (study and control). The study group including 150 anemic women divided into three subgroups (mild, moderate and severe anemic).

In the current study, the age of the patients in the two studied groups was matched without significant difference, the age in control group was 22-35 years; while in anemic group, it was 22-35 years.Regarding parity in both group, it ranged from 0-3.There was no significant difference between the two groups regarding parity. Gestational age at Doppler in both groups was ranged from 28-40 weeks withoutany significant difference between the two groups. The two studied groups must be matched regarding the demographic and basic maternal data to exclude the effect of age on outcomes.

In this study, hemoglobin level in anemic patients ranged from 5.0-10.9 g/dl ; while in non-anemic cases, it was 11-13.8 $\mathrm{g} / \mathrm{dl}$. There was a significant decrease in hemoglobin level in anemic more than non-anemic, these resultsmeet the inclusion and exclusion criteria.

In this study, it was found that middle cerebral artery resistance index in control group ranged from 0.792-0.923, in mild anemia group ranged from $0.78-0.98$, in moderate anemia group ranged from 0.79-0.91, in severe anemia group ranged from $0.56-0.80$. There was statistical significant relation between the different studied groups regarding middle cerebral artery resistance index $(P<0.05)$.

Middle cerebral artery pulsatility index in control group ranged from 1.42-1.71, in mild anemia group ranged from 1.15-1.75, in moderate anemia group ranged from 1.13-1.71, in severe anemia group ranged 
from 1.40-1.84. There was statistical significant relation between the different studied groups regarding middle cerebral artery pulsatility index $(P<0.05)$.

Middle cerebral artery systolic/diastolic ratio in control group ranged from 2.99-4.81, in mild anemia group ranged from $2.79-4.77$, in moderate anemia group ranged from 2.77-4.70, in severe anemia group ranged from 2.61-4.17. There was statistical significant relation between the different studied groups regarding middle cerebral artery systolic/diastolic ratio $(P<0.05)$.

In the control group, middle cerebral artery peak systolic velocity ranged from 31-51, in mild anemia group ranged from 31.7-50.60, in moderate anemia group ranged from 30.30-50.50, in severe anemia group ranged from 30-52. There was no statistical significant relation between the different studied groups regarding Middle cerebral artery peak systolic velocity $(P>0.05)$.

In this study, the umbilical artery resistance index in control group ranged from $0.50-0.68$, in mild anemia group ranged from $0.54-0.71$, in moderate anemia group ranged from $0.59-0.74$ and in severe anemia group ranged from $0.60-0.77$. There was a statistical significant relation between the different studied groups regarding umbilical artery resistance index $(P<0.05)$. Umbilical artery pulsatility index in control group ranged from $0.77-0.85$, in mild anemia group ranged from $0.60-0.88$, in moderate anemia group ranged from $0.67-0.90$, in severe anemia group ranged from $0.77-1.80$. There was statistically significant relation between the different studied groups regarding umbilical artery pulsatility index $(P<0.05)$.

Umbilical artery systolic/diastolic ratio in control group ranged from 2.09-2.2, in mild anemia group ranged from 2.13-2.23 with mean value, in moderate anemia group ranged from 2.10-3.40 and in severe anemia group ranged from 2.15-4.30. There was statistically significant relation between the different studied groups regarding Umbilical artery systolic/diastolic ratio $(P<0.05)$.

Middle cerebral/umbilical artery resistance ratio in control group ranged from $0.631-0.73$, in mild anemia group ranged from $0.692-0.724$, in moderate anemia group ranged from $0.746-0.813$ and in severe anemia group ranged from 0.96-1.07. There was statistical significant relation between the different studied groups regarding middle cerebral/umbilical artery resistance ratio $(P<0.05)$.

Maternal anemia is suspected to markedly reduce the oxygen supply to the fetus, which may be responsible for fetal blood flow redistribution, despite there is no evidence of placental insufficiency ${ }^{[7]}$. It is also suspected to reduce the oxygen supply to the growing fetus, leading to the redistribution of fetal blood flow. In the presence of fetal hypoxemia, fetal blood flow becomes centrally distributed to preserve cerebral oxygenation, known as the brain-sparing reflex; this plays a major role in fetal adaptations to oxygen deprivation. Maternal anemia is a hypoxic condition that could be responsible for the redistribution of fetal blood flow; however, no evidence of placental insufficiency has been documented currently. The combination of increased placental resistance and decreased cerebral resistance, measured using Doppler ultrasonography, is quantified by calculating the cerebral-to-umbilical artery resistance ratio. This Doppler parameter is not influenced by the duration of a pregnancy and is always greater than 1.1 during normal pregnancy ${ }^{[5]}$. However, this ratio is decreased in the presence of hypoxia due to increased placental resistance and cerebral vasodilatation ${ }^{[5]}$ and correlates closely with fetal growth, hypoxia, and behavior, particularly prior to 34 weeks of pregnancy ${ }^{[8]}$.

In moiety study, as for the $\mathrm{C} \backslash \mathrm{U}$ ratio, in groups $\mathrm{B}$ and $\mathrm{C}$ [moderate and severe anemia], the $\mathrm{C} / \mathrm{U}$ values were below the normal range $(<1.1)$, which confirms that the fetus had to adapt by increasing its blood flow redistribution towards the brain. Such adaptation was confirmed by the increase of the $\mathrm{C} / \mathrm{U}$ ratio after maternal red blood cell transfusion and parenteral iron supplementation. The increase in cerebral resistance after the transfusion without significant change in umbilical or uterine resistance confirms that maternal anemia does not create placental dysfunction and that the situation can be restored quickly by two units of red blood transfusion to the patient or intravenous iron. In Group (A) [mild anemia], the $\mathrm{C} / \mathrm{U}$ values were within the normal range, which means that the blood flow distribution between the brain and placenta was normal, despite the maternal hemoglobin content being significantly lower compared to normal ${ }^{[9]}$.

When the middle cerebral artery resistance index of the anemia group at admission was compared with the same group after 4 weeks of treatment, a significant increase was observed. This change could be the result of severe anemia-induced fetal cerebral vasodilatation being corrected following treatment. In comparison with the control group, a significantly lower middle cerebral artery pulsatility index was observed in the anemia group at admission, demonstrating that the cerebral adaptations observed were similar to those observed in fetuses experiencing anemia during pregnancies complicated by red cell alloimmunization ${ }^{[11]}$. However, fetal cerebral hemodynamics has been demonstrated to recover quickly following treatment for fetal anemia ${ }^{[12]}$.

In this current study, the results were in agreement with the study carried out by Ali et al. ${ }^{[13]}$, who stated that fetal Doppler examination at admission found that severe maternal anemia induced significant hemodynamic changes at the cerebral level. The mean middle cerebral artery resistance index was significantly lower in the anemia group than in the control group. This could be due to hypoxia-induced cerebral vasodilatation affecting the fetus during severeanemia ${ }^{[10]}$.

At admission, the umbilical artery resistance index 
was significantly higher in the anemia group. In hypoxic situations, the umbilical artery resistance index increases owing to an increase in placental resistance and the middle cerebral artery resistance index decreases owing to vasodilation. However, no significant differences were observed between the two patient groups in the umbilical artery pulsatility index and umbilical artery systolic/ diastolic ratio. The middle cerebral/umbilical artery resistance ratio cut-off value for predicting perinatal outcomes is 1.1 at $25-40$ weeks of pregnancy ${ }^{[10]}$. Measuring this parameter simplifies the quantification of fetal blood flow redistribution.

In Ali et al. ${ }^{[13]}$ study, the middle cerebral/umbilical artery resistance ratio was significantly lower at admission in the anemia group, suggesting that the fetus had adapted to anemic conditions by redistributing blood flow towards brain. The middle cerebral/umbilical artery resistance ratio in the anemia group increased significantly between admission and 4weeks as well as between admission and 6weeks. Severe maternal anemia results in the fetus experiencing a hypoxic state and this triggers a marked fetal cerebral vasodilatation to maintain oxygenation of brain tissue, which was evident in the present study by the observed decrease in the middle cerebral artery resistance index. Long-standing cerebral vasodilatation can result in a fetus experiencing cerebral edema and brain damage.

In this study, it was found that the neonatal ICU admission was significantly increased in severe anemic patients more than the mild and moderate anemic patients. In control and mild anemic groups, only 2 cases $(4.0 \%)$ of neonatal admitted to ICU; while in moderate anemic group, the number increased to double (4 cases) $8.0 \%$. In severe anemic group, the number of neonatal ICU admission was 9 cases $(18.0 \%)$, there was a significant increase in number of neonatal ICU admission in severe cases more than other groups $(P<0.05)$.

In agreement with this study, Rani et al. ${ }^{[14]}$ found that poor APGAR score (taken as score less than 7 at 5 minutes), early neonatal death and NICU admission were higher in anemic group than non-anemic group and it was statistically significant. This is in agreement with many studies ${ }^{[15,16]}$

The study done by Ghimire showed perinatal death 5\% in non-anemic group and $11 \%$ in severely anemic group. The present study also showed early neonatal death 3.08\% in non-anemic group and $10.08 \%$ in severely anemic group $^{[17]}$.

Kidanto et al. found that the risk of preterm delivery and low birth weight were significantly increased with the severity of anemia ; but in contrast to our study, they found no association between severity of anemia and APGAR score and early neonatal deaths ${ }^{[18]}$.

\section{CONCLUSION}

In conclusion, it was found that there was a significant effect of maternal anemia on fetal Doppler indices in both umbilical artery and middle cerebral artery in the last trimester of pregnancy. The increase in the severity of anemia decreases the cerebral lumbilical artery resistance index. Also,the study showed the severe anemia affect on neonatal ICU admission.

\section{CONFLICT OF ITNEREST}

There are no conflicts of interest.

\section{REFERENCES}

1. McLean E, Cogswell M, Egli I (2009): Worldwide prevalence of anaemia, WHO Vitamin and Mineral Nutrition Information System,1993-2005. Public Health Nutr., 12: 444-54.

2. Van Meter K (2008):A systematic review of the application of hyperbaric oxygen in the treatment of severe anemia. Undersea Hyper Med., 32 (1): 61-83.

3. Kalantar-Zadeh K, Block G, McAllister CJ, Humphreys MH, Kopple JD (2004): The American Journal of Clinical Nutrition, 80(2): 299-307.

4. Karaoglu L, Pehlivan E, Egri M (2010): The prevalence of nutritional anemia in pregnancy in an east Anatolian province, Turkey. BMC Public Health, 10: 329.

5. Christopher V and Charles S (2012): Iron Deficiency Anemia: A Public Health Problem of Global Proportions, Public Health - Methodology, Environmental and Systems Issues, Prof. Jay Maddock (Ed.), https://www.intechopen.com/.../ public-health-methodology-environmentaland-syste

6. WHO (2014): Guideline: Daily Iron and Folic Acid Supplementation in Pregnant Women. Geneva: World Health Organization. apps.who.int/iris/bitstream/10665/204712/ 1/9789241549523 eng.pdf

7. Divakaran TG, Waugh J, Clark TJ et al. (2001): Noninvasive techniques to detect fetal anemia due to red blood cell alloimmunization: a systematic review. Obstetrics and Gynecology, 98(3): 509-517.

8. Gramellini D, Folli MC, Raboni S, Vadora E, Merialdi A et al. (1992): Cerebral umbilical 
Doppler ratio as a predictor of adverse perinatal outcome. Cerebral and umbilical ratio: Obstet Gynecol., 79:416-20.

9. Moiety G and Ahmed Y (2012): Health care professionals, teachers, students and information professionals Arab World rely on Medical journal of Cairo university as a trusted source.The Medical Journal of Cairo University, 80(2): 15-35.

10. Carles G, Tobal N, Raynal P et al. (2003): Doppler assessment of the fetal cerebral hemodynamic response to moderate or severe maternal anemia. American Journal of Obstetrics and Gynecology, 188(3): 794-799.

11. Khalafallah A, Dennis A, Bates J et al. (2010): A prospective randomized, controlled trial of intravenous versus oral iron for moderate iron deficiency anaemia of pregnancy. Journal of Internal Medicine, 268(3): 286-295.

12. Vyas S, Nicolaides KH, Campbell S (1990): Doppler examination of the middle cerebral artery in anemic fetuses. American Journal of Obstetrics and Gynecology, 162(4): 1066-1068.

13. Ali E, Kumar M, Naqvi SE et al. (2016): Fetal vascular adaptation before and after treatment of severe maternal anemia in pregnancy. International
Journal of Gynecology and Obstetrics, 133(3): 284-286.

14. Rani KU, Gupta J, Gupta R et al.(2017): Maternal anaemia and its severity: an independent risk factor for preterm delivery and adverse neonatal outcome.International Journal of Reproduction, Contraception, Obstetrics and Gynecology, 3(2): 325-329.

15. Bakhtiar UJ, Khan Y, Nasar R (2007): Relationship between maternal hemoglobin and perinatal outcome. Age, 25: 24.

16. Mahamuda B, Tanira S, Feroza W et al. (2011): Effects of maternal anaemia on neonatal outcome a study done in the specialized urban hospital set up in Bangladesh. Bangladesh Journal of Medical Science, 10(3): 177-180.

17. Ghimire RH and Ghimire S (2013): Maternal And Fetal Outcome Following Severe Anaemia In Pregnancy: Results From Nobel Medical College Teaching Hospital, Biratnagar, Nepal. Journal of Nobel Medical College, 2(1): 22-26.

18. Kidanto HL, Mogren I, Lindmark G et al. (2009): Risks for preterm delivery and low birth weight are independently increased by severity of maternal anaemia. SAMJ: South African Medical Journal, 99(2): 98-102 